\section{Popova I., Mayboroda 0., Simurova $\mathbf{N}$., Karmashov 0.}

\title{
THE ANALISIS OF INTERACTION OF MONOSACCHARIDES WITH AMINOACIDS IN FOOD RAW BY OUANTUM-CHEMICAL METHODS
}

The object of research is the products of hydrolysis of the polysaccharide inulin (glucose, fructose) of plant raw materials used for the production of food products for health-improving and prophylactic purposes. The search for optimal conditions for the transformation of polysaccharides in the processes of hydrolytic cleavage into fructose-oligosaccharide products requires a thorough study of both the chemical composition of the raw material and the interaction between the components. This affects both the stability of the initial, intermediate and final products of the hydrolysis of the polysaccharide, and its release from plant materials and the further course of its fragmentation. Literature data indicate that the chemical composition of carbohydrate-containing plant materials, in particular the content of mineral components, has not yet been fully studied and requires additional research and refinement. After all, macro- and microelements in its composition are not only important nutrients, but can also take an active part in the transformation of organic components through complexation at intermediate stages of these processes. In addition, the question of the possible interaction of organic compounds in the composition of food raw materials, in particular biopolymers, which make up a significant part of the mass - carbohydrates and protein compounds, also requires attention. Such interaction under normal conditions has practically not been studied, but it can affect the course of technological processes of processing. The study of intermolecular interactions occurring in complex natural systems is often complicated either by the absence of direct (selective) physical and physicochemical research methods, or by the multicomponent chemical composition of the system, or by the complexity of the objects (substances) of the study themselves. This is especially true for natural substances of a polymeric nature - proteins, peptides, poly-and oligosaccharides. Therefore, the work paid special attention to the study of the interaction of these components. The spatial structure of inulin molecules, oligosaccharides and elementary units of these polymers has been investigated using quantum chemical modeling. The distribution of effective charges on carbohydrate atoms is calculated; it directly affects their reactivity. And also quantum-chemical models of the interaction of protein substances of plant materials with carbohydrates in vacuum and in aqueous solutions are created, depending on their dilution.

Keywords: polysaccharide hydrolysis, oligosaccharides, amino acids, quantum-chemical modeling, effective charges, sweeteners, chemical bonds.

Received date: 06.08.2020

Accepted date: 15.09.2020

Published date: 31.12 .2020
Copyright (C) 2020, Popova I., Mayboroda O., Simurova N., Karmashov O. This is an open access article under the CC BY license (http://creativecommons.org/licenses/by/4.0)

\section{Introduction}

The most common sugar substitutes for the carbohydrate group, which are mass produced and used in the food industry in many countries, are glucose-fructose syrups. Typically, such products are designated by the abbreviation GFS. Accordingly, glucose syrups with a high fructose content, or high-fructose syrups, are abbreviated as GFS. Depending on the fructose content, these syrups are sometimes referred to as first, second and third generation GFS.

Recently, the development of methods for the production of products of partial hydrolysis of inulin and mystic raw materials has been spreading; it has a number of advantages over the production of pure fructose based on the same inulin products $[1,2]$.

Known introduced effective technologies based on the hydrolysis of inulin, developed at the intersection of various sciences - physics, chemistry, biology, enzymology. Let's believe that such a technology requires a thorough study of the physical and chemical characteristics of inulin, its structure, in particular the spatial structure, as well as the use of modern mathematical and quantum-chemical apparatus, since at the present stage of development of scientific research, the development of technology is impossible without a preliminary study of the theoretical aspects of the structure and properties of the compounds involved in the technological process.

In addition, the question of the possible interaction of organic compounds in the composition of plant food raw materials, in particular biopolymers, which make up a significant part of carbohydrates and protein compounds, also requires attention. Such interaction under normal conditions has practically not been studied, but it can affect the course of technological processes of processing in hydrocarbonaceous raw materials.

In the scientific literature to designate products containing or consisting of inulin polymer homologues of a lesser 
degree of polymerization; there are no uniform approaches to terminology. Some authors use the generalized name for polysaccharides, the polymer chain of which consists of fructose - «fructan» residues. But this name does not carry information about the cyclic and anomeric forms of fructose (for example, $\beta$-D-fructofuranose, $\beta$-D-fructopyranose), or the type of glycosidic bonds between these residues, or the length of the polymer chain. For example, in the molecules of inulin and its polyhomologous between $\beta$-D-fructofuranose residues, a $1 \rightarrow 2$ glycosidic bond is realized, while in the levan molecule $2 \rightarrow 6$ there is a glycosidic bond, but both Lebanon and inulin are fructan [3, 4].

Therefore, it is relevant to study the interaction of individual chemical components of raw materials. Thus, the object of research is the hydrolysis products of the polysaccharide inulin (glucose, fructose) of plant raw materials used for the production of food products for health-improving and prophylactic purposes. And the aim of the work is to analyze and study the interaction of recombinations of polymer homologous inulin with a lesser degree of polymerization with aminoacetic acid.

\section{Methods of research}

The study of intermolecular interactions occurring in complex natural systems is often complicated either by the absence of direct (selective) physical and physicochemical research methods, or by the multicomponent chemical composition of the system, or by the complexity of the objects (substances) of the study themselves. This is especially true for natural substances of a polymeric nature - proteins, peptides, poly- and oligosaccharides. Therefore, recently, for the study of such objects, the methods of quantum-chemical modeling based on the solution of the Schrödinger equation for molecular systems have been successfully used. This takes into account all possible interactions that can occur between the nuclei and electrons of atoms, which formed a connection. As a result of quantum-chemical calculations, obtaining the spatial structure (arrangement of atoms relative to each other) and physical characteristics of the system (distances between atoms, angles, bond energies, enthalpy of formation) in the local energy minimum [5].

For quantum-mechanical modeling of the processes of possible interaction of carbohydrates, which are the constituent parts of inulin, a set of specialized computer programs «HyperChem» version 7.7 was used.

The first step of quantum chemical modeling was carried out using the semiempirical method MNDO (Modified Neglect ot Diatomic Overlap - modified neglect of diatomic overlap) in the parameterization PM3 (Parametric method Number 3), which allows, along with geometry optimization, to calculate the distribution of effective electrostatic charges on atoms included in molecule composition. In this case, the main attention was paid to the value of effective charges on oxygen and hydrogen atoms of hydroxyl groups as potential centers of interaction for the formation of hydrogen bonds [6, 7].

Since in the method of molecular mechanics atoms are considered as Newtonian particles interacting with each other due to potential fields (in this case, electrostatic), the potential interaction energy depends on the bond lengths, bond angles, torsion angles and non-covalent interactions. Therefore, the working force field (potential function) for calculations was chosen by the AMBER method (one with the methods of molecular mechanics for modeling the dynamics of organic molecules), focused on organic molecules and amino acids [8-10].

For the geometry of the system optimized by the method of molecular mechanics, the possibility of the formation of hydrogen bonds and their energy were calculated.

Since the interaction in real natural systems occurs mainly in aqueous solutions, where the solvent has a significant effect on the course of the processes, it was necessary to take into account the possible effect of water on intermolecular interactions in the monosaccharide-aminoacetic acid system. Therefore, the system of molecules optimized as described above was placed in a periodic box (conventional unit volume), uniformly filled with water molecules (from 50 to 700 molecules). Water dipoles with their own electrostatic fields affect the molecules of the solute (solvation interactions, dispersion interactions, etc.). Next, the geometry of the monosaccharide-amino acid-water system was optimized. After that, the calculation of hydrogen bonds was again carried out, provided that the minimum length between the solvent molecules and the solvated atoms should be $2.3 \AA$ [11].

\section{Research results and discussion}

When modeling the possible interaction of saccharides with aminoacetic acid with the formation of associates linked by hydrogen bonds, it was necessary to solve the following issues:

- which of the hydroxyl groups are actually involved

in the formation of hydrogen bonds;

- which of the amino acid groups is carboxyl or amino

to form hydrogen bonds with the alcohol groups of saccharides;

- which of the created connections will be strong.

At the first stage of quantum mechanical modeling, it was necessary to solve the problem of the most probable structure of inulin molecules in the solid state and in solution.

Since the synthesis of polysaccharides in plants occurs through a series of sequential polycondensation reactions, when the molecule grows due to the addition of the residue to the base monosaccharides, the molecular growth method was chosen for modeling. The essence of the method was that a fructose residue was attached to the sucrose molecule and the search for the most stable geometry was carried out for the resulting molecule. The resulting optimized geometry was used as a basis for attaching the next fructose molecule, and the optimized geometry was searched again. The extension was continued until 20 fructose residues entered the model molecule.

Calculations have shown that with the gradual growth of the molecule from 1 to $6-8$ fructose residues, a helical structure is predominantly formed. The resulting molecule is stabilized due to hydrogen bonds formed between the hydrogen atoms of mainly primary hydroxyl groups of fructose and etheric oxygen atoms, which bind the residues of fructose (Fig. 1).

Further extension of the chain by fructose residues leads to an even greater complication of the chain geometry and begins to turn into globular.

From the results obtained, it can be assumed that a further increase in the amount of fructose residues in the molecule will increase the globularity of the geometry. Thus, a strictly ordered geometry can't be expected for solid inulin. However, as is known, the geometry of complex 
polymer molecules in solution can change due to interaction with solvent molecules (solvation) and due to electrostatic influence on internal molecular hydrogen bonds (dispersion interactions). Therefore, the optimization of the geometry of the molecule with 20 fructose residues placed in an aqueous medium was carried out. The calculation results are shown in Fig. 2.

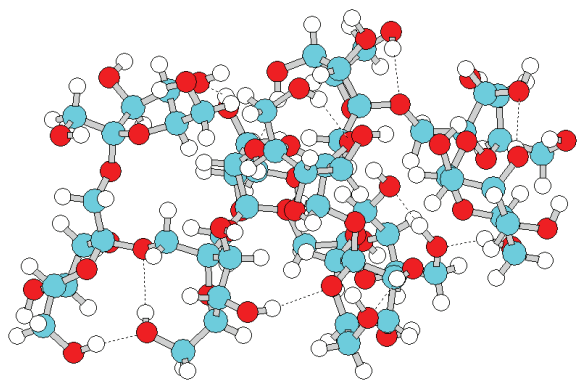

Fig. 1. Optimized geometry of the oligosaccharide molecule with 8 extended fructose residues

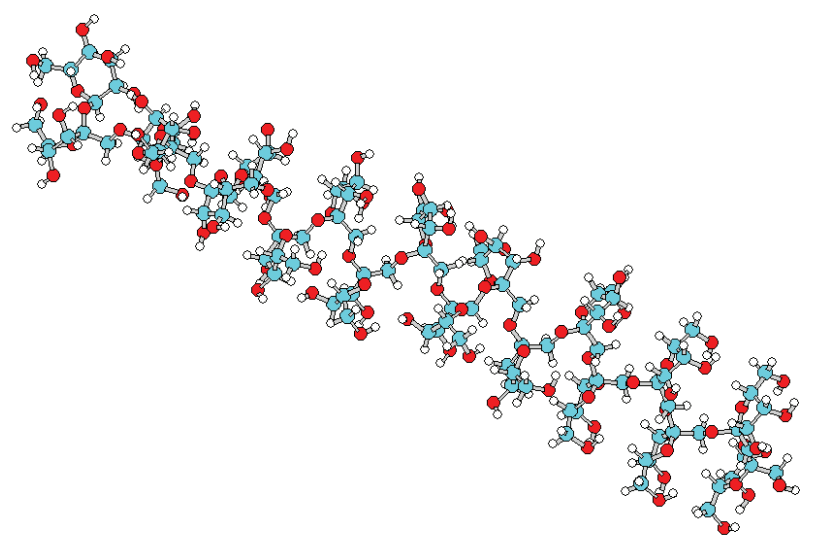

Fig. 2. Optimized geometry of the oligosaccharide molecule with 20 added fructose residues in a diluted aqueous solution

As can be seen from the results obtained in Fig. 2, when the oligosaccharide molecule passes into an aqueous solution, the globular structure gradually turns into a spiral structure. Moreover, the more diluted the solution, the less stable the globular structure becomes.

The strategy of quantum-chemical modeling in this work consisted in the Stepwise approximation of the system from vacuum-optimized geometries (optimized spatial structure) of saccharides ( $\alpha$-D-glucopyranose, $\alpha$-D-fructofuranose, $\beta$-D-fructofuranose, sucrose and raffinose) and aminoacetic acid, in vacuum-optimized system geometries. The resulting models simultaneously contained saccharide and amino acid molecules and were then transferred to an aqueous solution.

When optimizing the geometry of monosaccharide-amino acid systems, the main attention was paid to the possibility of the formation of hydrogen bonds, their number and strength, depending on the type of hydroxyl group of the saccharide and the orientation of the amino acid molecule relative to it. Taking into account the fact that the formation of hydrogen bonds is initially determined by electrostatic interactions, the molecular mechanics method was used to optimize the geometry of monosaccharide-amino acid systems, based on the classical Newtonian method for calculating the energy of the equilibrium geometry and molecular dynamics of an object.
Taking into account the fact that for hydroxyl groups it is possible to rotate around a single $\mathrm{C}$-O bond to create several conformations, and this can affect the distribution of effective charges on atoms in a molecule, geometry optimization was carried out for various initial hydrocarbon conformations. As shown by the calculations, a change in conformation affects the absolute value of the effective charge on a particular atom. However, in this case, the general trend of the distribution of charges over the atoms of the carbon molecule remains. So, in the case of glucose, the most negative charges are concentrated on the oxygen atoms directly associated with the pyranose cycle, the least charge on the oxygen of the primary hydroxyl group. At the same time, among the hydroxyl group of pyranose cycle, high charges prevail on oxygen of the acetal hydroxyl and oxygen with $C I=14$ (the so-called 1 - and 4-positions). The results are shown in Tables 1, 2 and in Fig. 3, $a, b$. This is perhaps a kind of confirmation of the fact that natural glucose-based polysaccharides predominantly bind through these positions. The data obtained suggest that, in the case of glucose, one should expect the formation of hydrogen bonds predominantly between these oxygen atoms and the hydrogen atoms of the protonated amino group of the acid. At the same time, the increased positive charge on the hydrogen atoms of the secondary hydroxyl groups will allow them to create hydrogen bonds with the carboxyl group of aminoacetic acid.

Table 1

Effective charges on oxygen and hydrogen atoms in the $\alpha$-D-glucopyranose molecule

\begin{tabular}{|c|c|c|}
\hline$C I$ & $T A$ & $Z_{e f}$ \\
\hline 1 & 0 & -0.283069 \\
\hline 13 & 0 & -0.317338 \\
\hline 14 & 0 & -0.318335 \\
\hline 15 & 0 & -0.301993 \\
\hline 16 & 0 & -0.344134 \\
\hline 18 & 0 & -0.310291 \\
\hline 20 & $\mathrm{H}$ & 0.210208 \\
\hline 21 & $\mathrm{H}$ & 0.207827 \\
\hline 22 & $\mathrm{H}$ & 0.201646 \\
\hline 24 & $\mathrm{H}$ & 0.213335 \\
\hline 23 & $\mathrm{H}$ & 0.197303 \\
\hline
\end{tabular}

Note: $C I$ - atom number; $T A$ - atom name; $Z_{e f}$ - effective charge

Table 2

Effective charges on oxygen and hydrogen atoms in the $\alpha$-D-fructofuranose molecule

\begin{tabular}{|c|c|c|}
\hline$C I$ & $T A$ & $Z_{e f}$ \\
\hline 1 & 0 & -0.295290 \\
\hline 11 & 0 & -0.294891 \\
\hline 12 & 0 & -0.304072 \\
\hline 13 & 0 & -0.303948 \\
\hline 14 & 0 & -0.302623 \\
\hline 22 & 0 & -0.303075 \\
\hline 20 & $\mathrm{H}$ & 0.196434 \\
\hline 21 & $\mathrm{H}$ & 0.200862 \\
\hline 22 & $\mathrm{H}$ & 0.213179 \\
\hline 24 & $\mathrm{H}$ & 0.189973 \\
\hline 23 & $\mathrm{H}$ & 0.194059 \\
\hline
\end{tabular}

Note: $C I$ - atom number; $T A$ - atom name; $Z_{\text {ef }}$ - effective charge 

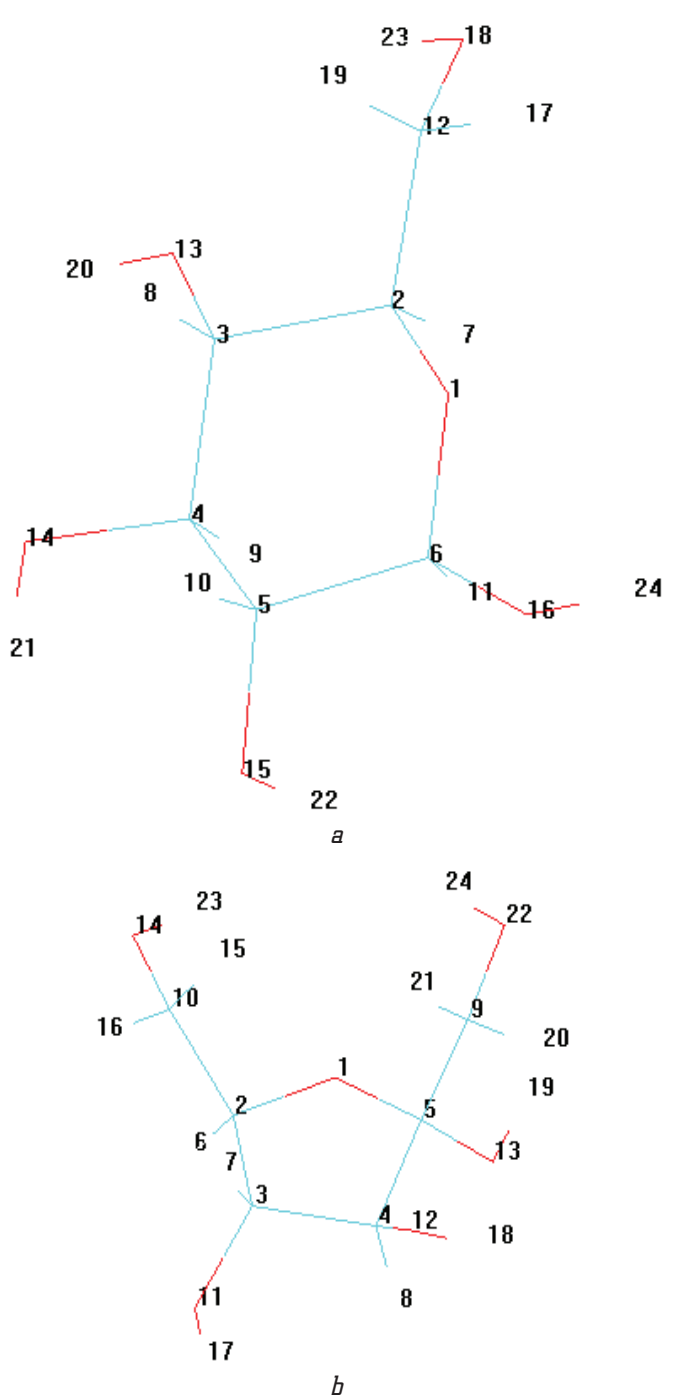

Fig. 3. Results of quantum-chemical optimization of the geometry of molecules: $a-\alpha$-D-glucopyranose; $b-\alpha$-D-fructofuranose; the numbers in the figure are the numbers of atoms for which the effective charges are calculated donor-acceptor (H-bond) - were calculated. Based on the obtained values, it can be concluded about the contribution of each component to the connection, and, accordingly, about the overall stability of the connection. Thus, in the case of glucose, the optimization of the geometry of the system confirmed the possibility of the formation of hydrogen bonds both through the carbonyl and amino groups of the acid (Fig. 4). In this case, the electrostatic and donor-acceptor components give contributions of the same order to the enthalpy of hydrogen bond formation both through the carboxyl group and through the amino group, although the electrostatic contribution is slightly larger.

With an arbitrary orientation of an amino acid, or with orientation of an amino acid to a glucose molecule of both carboxyl and amino groups, optimization of the geometry of the system showed that the formation of several hydrogen bonds at the same time is more advantageous. In this case, the electrostatic contribution to the hydrogen bond energy increases by almost an order of magnitude. A similar result is observed if the geometry of the system is optimized, which includes both a monosaccharide molecule and two amino acid molecules.

When replacing glucose with other saccharides, it does not significantly change the results of geometry optimization the formation of several hydrogen bonds remains more advantageous and the contribution of the electrostatic component clearly exceeds the contribution of the donor-acceptor interaction. The latter conclusion is essential in the transition from calculating chemical systems in vacuum to optimizing the geometry of chemical systems in aqueous solutions.

Since an aqueous medium can simultaneously perform two functions, chemical (solvating) and physical (dispersing, due to weakening of electrostatic interaction), it is necessary to determine the effect of water molecules on the stability of multicenter hydrogen bonds.

It is logical to assume that hydrogen bonds, the electrostatic contribution of which is the greatest, should weaken or even completely break in an aqueous medium. At the same time, the groups with a higher contribution of the donor-acceptor component little feel the influence of the environment and will be stored in solutions.
In the case of $\alpha$-D-fructofuranose and $\beta$-D-fructofuranose, the effective charges on the oxygen atoms of the hydroxy groups turned out to be practically the same, while the oxygen of the furanose cycle has the lowest effective charge. At the same time, the greatest positive charge on hydrogen is concentrated mainly on the acetal hydroxyl.

To solve the above issues, a stepwise approximation approach was chosen, which consisted in the following. The amino acid molecule in the form of a dipolar ion is forcedly oriented by the corresponding carboxyl or amino group to each of the alcohol groups of the corresponding carbon. The geometry of the resulting system was optimized by searching for a common local minimum in the energies of hydrogen bond formation, provided that the minimum bond length should not exceed $2.3 \AA$. For the obtained optimized geometry of the system, the components of the hydrogen bond energy - electrostatic and

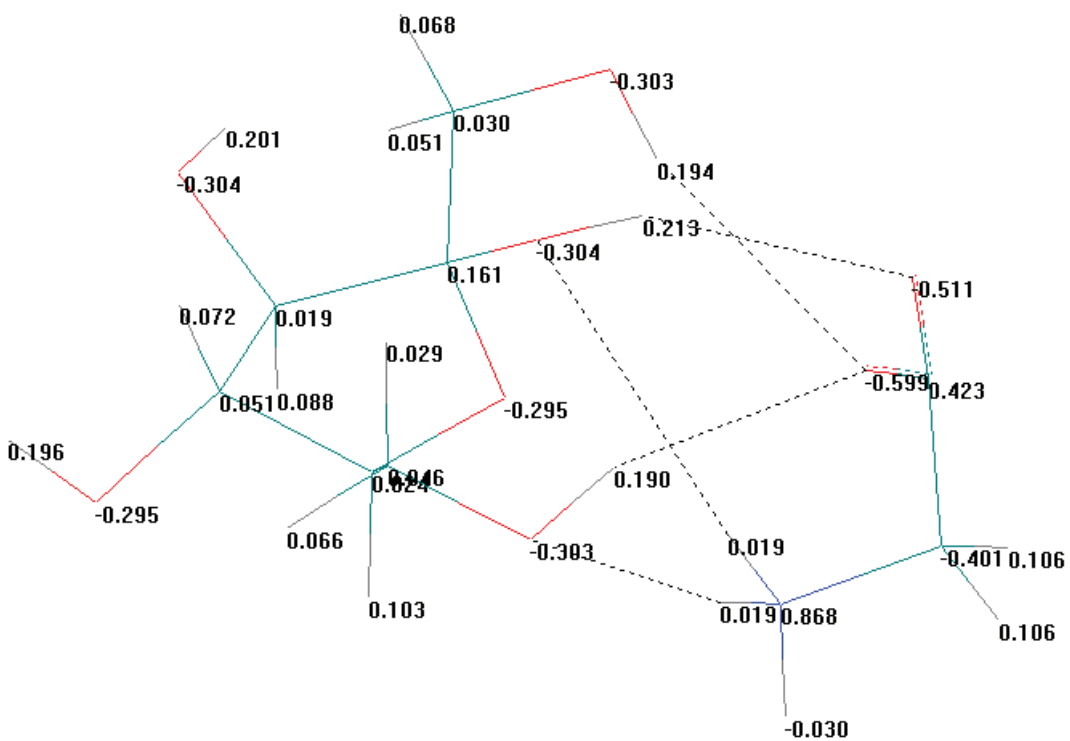

Fig. 4. Results of optimization of the geometry of the glucose-aminoacetic acid system by molecular mechanics methods for the initial orientation of the amino acid to the fructose molecule through the carboxyl and amino groups simultaneously: H-bond =-0.132845; Electrostatic $=-35.6097$ 
To confirm this assumption, calculations were performed to optimize the geometry of the saccharide-aminoacetic acid system in aqueous solutions. For this, the monosaccharideaminoacetic acid associates with the geometry optimized in vacuum were placed in a periodic box filled with water molecules, and the geometry of the conventional solution was optimized. For the obtained optimized model, let's additionally calculate all possible hydrogen bonds in the system.

To take into account the possible effect of the concentration of dissolved components (that is, the number of water molecules in a unit of a conventional aqueous solution) on the stability of hydrogen bonds, the calculation of optimized geometries and hydrogen bonds in the system was carried out for two periodic boxes of different volume. The volume of the first contained at least 50 water molecules in a periodic box, which is typical for concentrated solutions. The second - at least 600 water molecules (conditionally diluted solution). Calculations for each system under the same initial conditions were performed at least 5 times. The results were considered adequate if they matched at least 3 times.

Some of the results obtained are shown in Fig. 5. For better visual perception, water molecules have been removed in most of the figures, which do not take part in the solvation processes of the constituent components of the system.

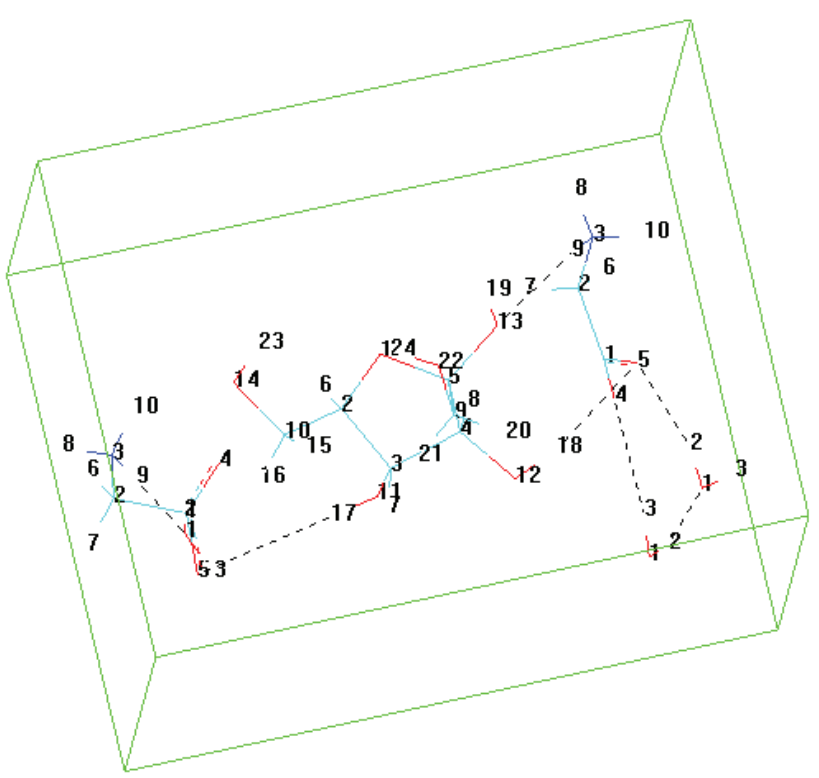

Fig. 5. Results of geometry optimization of the $\alpha$-D-fructofuranoseaminoacetic acid system (ratio 1:2) in a concentrated aqueous solution

The alcohol groups of the monosaccharide are also partially hydrated. An interesting fact is that the reproducibility of hydration of alcohol groups according to the results of calculations is not high. This may indicate the dynamic nature of hydration.

\section{Conclusions}

The results indicate that in the case of saccharides, especially with an increase in the number of their monomeric units, the distribution of effective charges is gradually leveled in comparison with the molecules of monosaccharides. This should lead to an even probable (statistical) formation of monosaccharide-amino acid associates due to hydrogen bonds. However, given the fact that with an increase in the number of monosaccharide elementary units in molecules, intramolecular self-organization processes (formation of helices, globules, etc.) can occur, then only a part of hydroxyl groups available for the formation of intermolecular associates is not included in self-organizing processes.

Based on the obtained results, it can be argued that: - hydration significantly affects the interaction between amino acid and monosaccharide;

- in aqueous solutions, one hydrogen bond is predominantly formed between the monosaccharide and the amino acid;

- probability of the formation of hydrogen bonds between the functional groups of the amino acid and the saccharide molecules is practically the same;

- in dilute solutions, the interaction between amino acid and monosaccharide is worse than in concentrated solutions;

- hydration of alcohol groups of the monosaccharide is dynamic.

A consequence of the above indicators may be the fact that the dynamic nature of the hydration of alcohol groups of the monosaccharide will lead to the appearance of vacant unsolvated hydroxyl groups. These groups will be a kind of active centers for the formation of $\mathrm{H}$-bonded associates between amino acid and monosaccharide molecules. At the same time, these centers will be of a wandering character and will form hydrogen bonds with functional groups of the amino acid with the same probability. Since the energies of solvation bonds and $\mathrm{H}$-bonds of amino acid-monosaccharide are almost the same, the formation and destruction of associates can continue for a rather long time, thereby leading to the cyclical nature of their change in the study of the polarimetric properties of systems.

\section{References}

1. Doronin, A. F., Ipatova, L. G., Kochetkova, A. A., Nechaev, A. P., Khurshudian, S. A., Shubina, O. G. (2009). Funktsionalnye pischevye produkty. Vvedenie v tekhnologii. Moscow: DeLi Print, 288.

2. Hager, C., Miethchen, R., Reinke, H. (2000). Epimerisation of Carbohydrates and Cyclitols, 17.1 Synthesis of Glycosyl Azides and N-Acetyl Glycosyl Amines of Rare Monosaccharides. Synthesis, 2000 (2), 226-232. doi: http://doi.org/10.1055/s-2000-6250

3. Wang, Q., Dordick, J. S., Linhardt, R. J. (2002). Synthesis and Application of Carbohydrate-Containing Polymers. Chemistry of Materials, 14 (8), 3232-3244. doi: http://doi.org/10.1021/ cm0200137

4. Cherepanov, I. S., Trubachev, A. V., Abdullina, G. M. (2016). Amino-karbonilnye vzaimodeistviia uglevodov s zameschennymi aromaticheskimi aminami. Khimicheskaia fizika i mezoskopiia, 18 (2), 310-315.

5. Tomasik, P. (2004). Chemical and functional properties of food saccharides. New York: CRC Press LLC, 399.

6. Jing, H., Kitts, D. D. (2004). Antioxidant activity of sugar-lysine Maillard reaction products in cell free and cell culture systems. Archives of Biochemistry and Biophysics, 429 (2), 154-163. doi: http://doi.org/10.1016/j.abb.2004.06.019

7. Solozhenkin, P. M., Solozhenkin, O. I., Krausz, S. (2012). Prediction of Efficiency of Flotation Collectors Based on Quantum Chemical Computations. Books of Abstracts. XXVI International Mineral Processing Congress-IMPC. New Delhi, 2, 638.

8. Solozhenkin, P. M. (2012). Quantum-chemical and moleculardynamic aspects of forecasting of properties of collectors of metals from productive solutions of nonferrous metals. Week of the miner 2012. Separate release of the mountain information analytical bulletin (scientific and technical magazine). Publishing house «Mountain book», 1, 431-455. 
9. Dunning, T. H. (1989). Gaussian basis sets for use in correlated molecular calculations. I. The atoms boron through neon and hydrogen. The Journal of Chemical Physics, 90 (2), 1007-1023. doi: http://doi.org/10.1063/1.456153

10. Becke, A. D. (1997). Density-functional thermochemistry. V. Systematic optimization of exchange-correlation functionals. The Journal of Chemical Physics, 107 (20), 8554-8560. doi: http:// doi.org/10.1063/1.475007

11. Neese, F. (2008). ORCA - an ab initio. Density Functional and Semiempirical program package. Version 2.9. University of Bonn. Available at: http://rossi.chemistry.uconn.edu/chem5326/ files/OrcaManual_2_9.pdf

Popova Inna, Doctor of Philosophy, PhD, Professor, Departmen of Food Chemistry, National University of Food Technologies, Kyiv,
Ukraine, e-mail: ivpopova@bigmir.net, ORCID: http://orcid.org/00000003-0332-2681

Mayboroda Olena, PhD, Associate Professor, Department of Food Chemistry, National University of Food Technologies, Kyiv, Ukraine, e-mail: mayboroda_l@rambler.ru, ORCID: http://orcid.org/0000$0002-8252-6393$

Simurova Natalia, PhD, Associate Professor, Department of Food Chemistry, National University of Food Technologies, Kyiv, Ukraine, e-mail: n.v.simurova@gmail.com,ORCID: http://orcid.org/00000003-0310-6153

Karmashov Oleksandr, Department of Food Chemistry, National University of Food Technologies, Kyiv, Ukraine, e-mail: n.v.simurova@gmail.com, ORCID: http://orcid.org/0000-0002-4841-3766

\section{Havriushenko K. Gladkiy F.}

\section{ANALYSIS OF THE ETHYL STEARATE PROPERTIES AS A NEW ALTERNATIVE TO COCOA BUTTER}

The object of research is the product of modification of fats, in particular derivatives of fatty acids and monohydric alcohols, namely ethyl stearate. One of the biggest challenges for the confectionery industry is the use of modified fats that fully meet food safety requirements. Existing modified fats, used in confectionery, mainly contain saturated fatty acids with carbon atoms of 16 or less, identified by the World Health Organization (WHO) as harmful to the human body. Without the presence of a significant amount of trans fatty acids, it is difficult to obtain inexpensive modified fats that have high hardness at a low melting point. The reduction of trans fatty acid isomers in modified fats is a worldwide problem. In addition, the presence of glycerol in the composition of trihydric alcohol fats can promote the formation of glycidol esters. In turn, glycidol esters are known to contribute to the development of cancer. It is practically impossible to obtain triacylglycerols, which contain only stearic acid among the saturated fatty acids. This is a significant obstacle to the formation of a rational fatty acid composition in terms of the content of saturated acids.

It is proposed to solve certain problems by means of a new technology for modifying fats, namely by replacing in the composition of fats - acylglycerol of the alkyl group. As an alternative to cocoa butter in confectionery products, according to the author's data, stearic acid ethyl ester can be used. A complex of studies has determined that ethyl stearate, by its physical and chemical properties, namely: melting point, mass fraction of solid ethers and solubility in acylglycerols in any ratio, is a full-fledged alternative to cocoa butter.

The use of new modified fats, in particular ethyl stearate in the composition of confectionery, will allow to exclude saturated fatty acids from the diet; according to WHO, they contribute to an increase in low density lipoproteins in human blood.

Keywords: fat and oil industry, modified fats, ethyl esters of fatty acids, stearic acid, confectionery industry.

\section{Introduction}

A promising direction in the fat and oil industry is the production of modified fats by changing the composition of the alkyl group, that is, the conversion of fatty acids into ethers of monohydric alcohols, in particular ethyl alcohol. In this way, it is easy to obtain modified fats with high hardness and low melting point without trans-isomers, as well as selectively regulate their fatty acid composition [1]. Full or partial replacement of fats in ethyl esters of fatty acids will allow creating compositions with a narrow range of melting points, hardness and other structural, mechanical and physicochemical properties, which is extremely important for the confectionery industry.

Difficulties in the creation of fats without trans fatty acid isomers (TFAI) lead to a decrease in demand for margarine products from consumer industries [2]. In many countries, regulation has been introduced for the TFAI content in finished products. For example, in Ukraine, at the legislative level, this indicator is limited in spreads (DSTU 4445:2005), sandwich margarines (DSTU 4465:2005) and minarines (DSTU 4564:2006) to $8 \%$. For other products regulating the TFAI content is absent [3]. The production of margarine is partially supported by modified fats with a minimum 